Sharif University of Technology
Scientia Iranica
SCIENTIA
I RAN I CA
http://scientiairanica.sharif.edu

\title{
Domain reduction method for seismic analysis of dam-foundation-fault system
}

\author{
H. Mohammadnezhad ${ }^{a}$, H. Zafarani ${ }^{b}$, and M. Ghaemian ${ }^{a, *}$ \\ a. Department of Civil Engineering, Sharif University of Technology, Tehran, P.O. Box 11155-9313, Iran. \\ b. International Institute of Earthquake Engineering and Seismology (IIEES), Tehran, P.O. Box 19395/3913, Iran. \\ Received 13 June 2016; received in revised form 19 February 2017; accepted 3 July 2017
}

\author{
KEYWORDS \\ Domain reduction \\ method; \\ Dam-foundation-fault \\ system; \\ Seismic wave \\ propagation; \\ Numerical method.
}

\begin{abstract}
Numerical simulation of dam-foundation-fault system, considering the earthquake source, propagation path, and local site effects, was carried out for realistic and reasonable seismic safety analysis of concrete dams. The Domain Reduction Method (DRM) was used for seismic analysis of Dam-Foundation-Fault (DFF) system, in which a modular two-step methodology for reducing the computational costs in large domain analysis was introduced. In this method, seismic excitation is directly applied to the computational domain such that assigning artificial boundary to the finite element models is more comfortable. In order to verify the implementation of the DRM in Finite Element Method (FEM), a simple 2D half-space under the Ricker wavelet excitation was examined. Then, to investigate the DRM as an appropriate method in seismic analysis of DFF system, the Koyna concrete gravity dam was modeled. Comparing the obtained results by using both the DRM in a small domain and the traditional approach in the large domain containing the source shows the efficiency of the DRM in terms of computational costs, such as running time and number of elements for seismic analysis of concrete gravity dams.
\end{abstract}

(C) 2019 Sharif University of Technology. All rights reserved.

\section{Introduction}

Concrete dams are among the most important infrastructures in every country and hence, their safety during severe earthquakes is extremely important. Reliable designing of concrete dams requires accurate simulation of the dam-foundation-reservoir system and choosing of suitable seismic excitation. The behavior of dam-foundation-reservoir system has been investigated extensively during recent years [1-7]. Dynamic analysis of dam-foundation-reservoir systems is a complex problem by nature. These systems consist of three domains

\footnotetext{
*. Corresponding author. Tel.: +982166164242;

Fax: +982166014828

E-mail address: ghaemian@sharif.edu (M. Ghaemian)
}

with different behaviors. In addition, foundation and reservoir have at least one infinite dimension, which would add to the complexity of the problem. Linear or nonlinear dynamic analysis of complex systems, such as dam-foundation-reservoir, is performed numerically. Numerical methods such as Finite Element (FE) and boundary element are common tools in damfoundation-reservoir system analysis.

Because of unique characteristics of the domains involved, numerical modeling of these domains, as well as properly defining the interactions between them, is a challenging work. Trying to overcome these challenges in order to obtain more realistic responses from the system is an invaluable effort. Because of superiority of finite element method over boundary element method in modeling complex and nonlinear environments, this method is widely used in the analysis of dam-foundation-reservoir system. 
Although valuable information has been achieved, some challenges still remain, including the interaction of dam-foundation-reservoir as well as estimation of the suitable excitation and its implementation in the numerical models.

One of the effective features in seismic analysis of dam-foundation systems is proper modeling of infinite massed foundation in the numerical model (especially in finite element method). To cut temporal and analytical costs, infinite foundation should be truncated at some designated artificial boundary. This artificial boundary is responsible for absorbing scattered waves emanating from the structure. Various types of artificial boundaries have been proposed by researchers during recent years [8-13].

Earthquake input mechanism is another source of dilemma in seismic analysis of these systems. Leger and Boughoufalah [14] studied the differences of various earthquake input mechanisms in finite element analysis of the dam-foundation-reservoir system. Boundary conditions and earthquake input mechanisms should be chosen in a way to be able to include the effects of wave propagation in semi-infinite foundation environment. These challenges are the main reasons the researchers tend to use massless foundation model, which was originally proposed by Clough [15]. Numerous studies show that massless model yields overestimated results $[16,17]$.

To take a further step in correct simulation of earthquakes for analysis of unique structures and infrastructures such as dams, bridges, etc., which are very important both socially and economically, other effective parameters such as site conditions and characteristics, existing faults in the region, probable scenarios for earthquakes, etc. should be included in the model. Many researchers have investigated the effects of different parameters of earthquakes on response of structures [18-22].

Proper estimation of the ground motion input for a structure requires a deep understanding and accurate modeling of fault mechanism, propagation path of the seismic wave, earthquake site effects, and the interaction of soil-structure. Each of these factors may affect the ground motion input as well as the structural response depending on the selected scenario.

In the field of earthquake simulation, some noteworthy studies have been published in recent years $[23,24,25]$. These studies are conducted in a semiinfinite environment with respect to all assumptions made for characteristics of the environment, fault, and rupture mechanisms.

One faces two different scales in dam-foundationfault problem. The problem of simulating fault and earthquake (seismological scale (kilometers)) and the problem of modeling dam-foundation-reservoir system (engineering scale (meters or centimeters)) should be properly combined so that by using features of both problems, more realistic and logical results are obtained.

There are two general approaches to considering these issues: direct and hybrid methods. In the direct method, all components of the fault-structure are simulated in one system, which includes a seismic source (usually represented by a shear crack or its body-force equivalents), a crustal model, near-surface soil model, and structures at the surface. Despite being straightforward and favorable, large dimensions of the fault model and propagation path (several kilometers) along with the required accuracy for response of the structure make the direct analysis time-consuming and less efficient from a computational resource point of view.

In the second approach, hybrid methods are used in order to solve this problem. These methods have acceptable accuracy with lower computational cost. Hybrid methods are performed in two steps. In the first step, the ground motion is computed by removing the structure and localized geological features. In the second step, only a small region of the domain is accurately modeled. The computed ground motion is utilized to determine the equivalent force which is applied in the second step.

Examples of hybrid methods can be found in several references, which have utilized different numerical and analytical methods at each step of the algorithm. Mossessian and Dravinski [26] investigated the problems of diffraction of plane harmonic $\mathrm{P}, \mathrm{SV}$, and Rayleigh waves by subsurface irregularities of arbitrary shape by combination of the finite element method with indirect boundary integral equation approach. Bielak et al. [27] developed a BEM-FEM hybrid method and used it to investigate semicircular inhomogeneous valleys with linearly increasing shear modulus with depth, due to oblique incident $\mathrm{SH}$ waves. Fäh et al. [28] simulated the ground motion in Mexico City caused by the Michoacan earthquake of September 19, 1985. They used a hybrid technique that was based on a combination of modal summation and the finite-difference method. Zahradnı'k and Moczo [29] developed a new hybrid method for treating the seismic wave fields at localized 2D near-surface structures excited by a point source. They combined the discretewave number and finite-difference methods. Moczo et al. [30] presented a generalization of the hybrid DW-FD method of Zahradnı'k and Moczo [29] for computation of P-SV seismic motion at inhomogeneous viscoelastic topographic structure. The method was based on a combination of the Discrete-Wavenumber (DW), Finite-Difference (FD), and Finite-Element (FE) methods.

In spite of the enormous computational progress in recent years $[31,32,33]$, estimating the seismic per- 
formance of a fault-structure system poses several challenges, such as applying the seismic wave to the numerical model, wide range of multiple spatial and temporal scales, heterogeneous model for soil and geological structure, and nonlinearity of soil. Creating an appropriate model and developing a numerical method to analyze them are the main challenges. To summarize, the following challenges are foreseen in the modeling of the dam-foundation-faults system:

1. Simulating fault and rupture mechanism in earth with acceptable scale;

2. Modeling wave propagation from fault to vicinity of the structure (near-field);

3. Modeling massed infinite foundation with finite element method;

4. Properly implementing earthquake in analytical model;

5. Assigning appropriate boundary conditions at truncated boundaries for absorption of scattered waves.

Bielak et al. [34] developed the DRM for seismic analysis and numerical simulation of large-scale problems including seismic source (i.e., fault), propagation path, and locally complex structures effects (e.g., strong geological or topographical irregularities). Yoshimura et al. [35] verified the domain reduction method using the Green function approach. They also demonstrated the application of the domain reduction method to three-dimensional problems with large computational domains, including faults and high heterogeneous mediums.

The DRM method is a two-step procedure through which the domain is reduced by changing the variable during the solution. Seismic excitation is directly applied to the computational domain and the virtual boundaries in the model (such as viscous boundaries) should only absorb the scattered energy radiated from the structure. The domain reduction method is also useful for investigating the interaction of soil-structure in which the fault and propagation path are not considered. The performance of domain reduction method using absorbing boundaries by modeling a tunnel has been assessed by Kontoe et al. [36]. Domain reduction method, with its special features, is a useful tool to overcome the mentioned challenges of the damfoundation-fault problem. In this study, a numerical framework based on the domain reduction method is proposed to investigate the dynamic response of the dam-foundation-fault system. The domain reduction method for analyzing the dam-foundation-fault system is verified by a simple model in the first step. The Koyna concrete gravity dam is analyzed using this method for the purpose of real-life structure analysis.

\section{The DRM concept}

The DRM is a two-step procedure, which reduces the computational domain by changing the variables of the dominant equations. In this method, seismic excitation is applied directly to the computational domain. The framework of the DRM is based on the study of Bielak et al. [34].

In the first step, seismic excitation is applied to the computational domain. During this step, displacements of the free-field and effective forces on a onedimensional strip are calculated and reserved. In the second step, the results of the first step are utilized to determine the equivalent force which is applied in the computational domain.

\subsection{Formulation of $D R M$}

The same formulation of DRM as the one by Bielak et al. [34] is used in this study. The general framework of the problem, including a semi-infinite medium with specific layering, fault as seismic source, and dam, is illustrated in Figure 1.

Reduction of the computational domain is desired due to costs and time-consuming nature of numerical methods. It is necessary to transfer the effects of excitation to the structure by minimum changes. The virtual boundary of $\Gamma$ breaks the total area into two parts; one includes site and structure, denoted by $\Omega$, and the other is infinite medium, denoted by $\Omega^{+}$, which includes the fault as well. In order to solve the main problem, the semi-infinite medium is limited to the $\Gamma^{+}$boundary. The vector of nodal displacements within the internal domain of $\Omega$, external domain of $\Omega^{+}$, and the boundary between them, $\Gamma$, is depicted in Figure 2 by $u_{i}$ (internal), $u_{e}$ (external), and $u_{b}$ (border), respectively. The whole domain is divided into two subdomains, including the fault (the far field), and structure and site (the near field), according to Figure 2. The displacement of $u_{b}$ on the border of $\Gamma$ is continuous and $P_{b}$ is the nodal forces which are transferred from $\Omega^{+}$to $\Omega$ (according to Figure 3 ).

Navier's equations are the dominant equations of

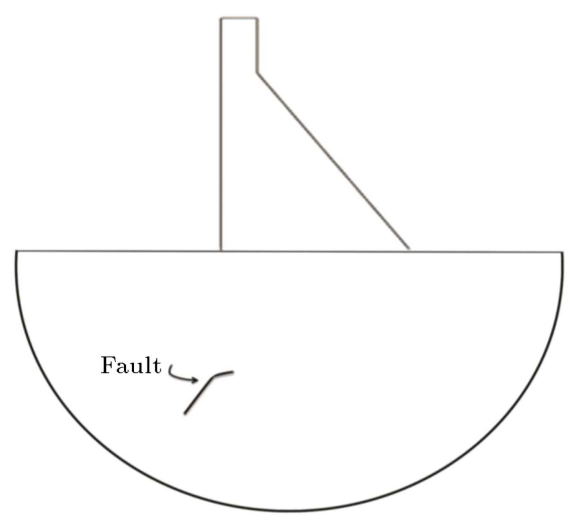

Figure 1. Schematic of dam-foundation-fault system. 


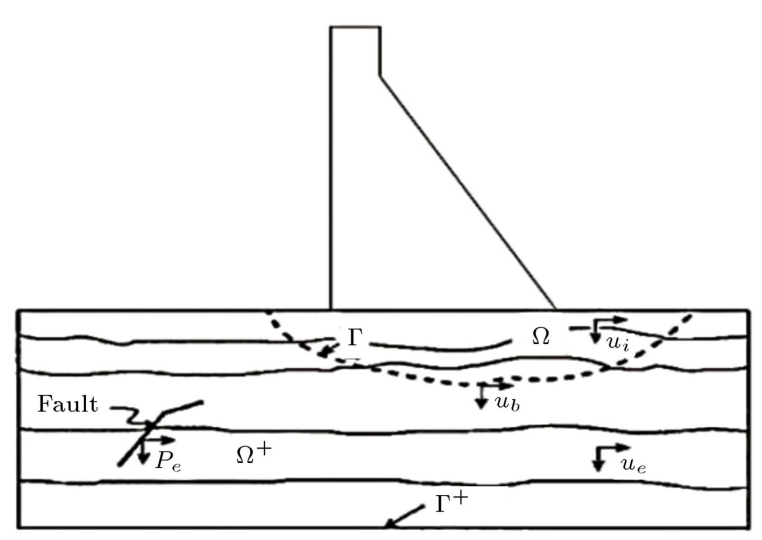

Figure 2. Truncated seismic region.

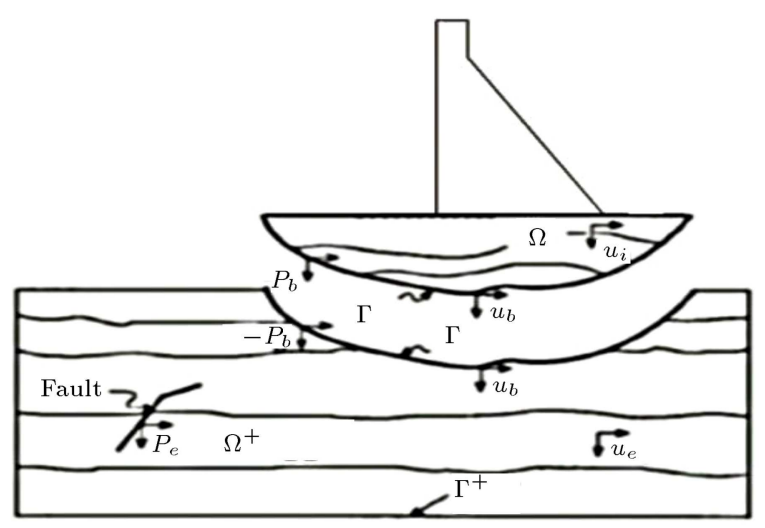

Figure 3. Regions partitioned into two subdomains.

motion in the entire domain. The mentioned equations for the $\Omega$ and $\Omega^{+}$domains are discretized using the FE method as follows:

$$
\left[\begin{array}{cc}
M_{i i}^{\Omega} & M_{i b}^{\Omega} \\
M_{b i}^{\Omega} & M_{b b}^{\Omega}
\end{array}\right]\left\{\begin{array}{c}
\ddot{u}_{i} \\
\ddot{u}_{b}
\end{array}\right\}+\left[\begin{array}{cc}
K_{i i}^{\Omega} & K_{i b}^{\Omega} \\
K_{b i}^{\Omega} & K_{b b}^{\Omega}
\end{array}\right]\left\{\begin{array}{c}
u_{i} \\
u_{b}
\end{array}\right\}=\left\{\begin{array}{c}
0 \\
P_{b}
\end{array}\right\},
$$

in $\Omega$,

$$
\left[\begin{array}{cc}
M_{b b}^{\Omega^{+}} & M_{b e}^{\Omega^{+}} \\
M_{e b}^{\Omega^{+}} & M_{e e}^{\Omega^{+}}
\end{array}\right]\left\{\begin{array}{l}
\ddot{u}_{b} \\
\ddot{u}_{e}
\end{array}\right\}+\left[\begin{array}{cc}
K_{b b}^{\Omega^{+}} & K_{b e}^{\Omega^{+}} \\
K_{e b}^{\Omega^{+}} & K_{e e}^{\Omega^{+}}
\end{array}\right]\left\{\begin{array}{l}
u_{b} \\
u_{e}
\end{array}\right\}=\left\{\begin{array}{c}
-P_{b} \\
P_{e}
\end{array}\right\}
$$

$$
\text { in } \Omega^{+} \text {, }
$$

where $M$ and $K$ represent mass and stiffness matrices, respectively; $i, e$, and $b$ indices represent the internal, external, and boundary nodes; and $\Omega$ and $\Omega^{+}$superscripts define the area to which the matrix belongs. By adding these equations, the usual equation of motion for the entire domain is derived as follows:

$$
\begin{array}{r}
{\left[\begin{array}{ccc}
M_{i i}^{\Omega} & M_{i b}^{\Omega} & 0 \\
M_{b i}^{\Omega} & M_{b b}^{\Omega}+M_{b b}^{\Omega^{+}} & M_{b e}^{\Omega^{+}} \\
0 & M_{e b}^{\Omega^{+}} & M_{e e}^{\Omega^{+}}
\end{array}\right]\left\{\begin{array}{l}
\ddot{u}_{i} \\
\ddot{u}_{b} \\
\ddot{u}_{e}
\end{array}\right\}} \\
+\left[\begin{array}{ccc}
K_{i i}^{\Omega} & K_{i b}^{\Omega} & 0 \\
K_{b i}^{\Omega} & K_{b b}^{\Omega}+K_{b b}^{\Omega^{+}} & K_{b e}^{\Omega^{+}} \\
0 & K_{e b}^{\Omega^{+}} & K_{e e}^{\Omega^{+}}
\end{array}\right]\left\{\begin{array}{l}
u_{i} \\
u_{b} \\
u_{e}
\end{array}\right\}=\left\{\begin{array}{c}
0 \\
0 \\
P_{e}
\end{array}\right\} \dot{(3)}
\end{array}
$$

In order to transfer the seismic excitation from the fault to the border of $\Gamma$, an auxiliary problem with a consistent external domain (including the fault) is utilized. The internal domain of $\Omega_{0}$ is simple, excluding the structure. Therefore, the background model induces the free-field of the main model when the structure and site conditions are neglected. Solving this problem is simpler than the main one. The nodal displacements and boundary forces are shown by $u_{i}^{0}$, $u_{b}^{0}, u_{e}^{0}$, and $P_{b}^{0}$, according to Figure 4 .

The equation of motion for $\Omega^{+}$in the auxiliary problem is according to Eq. (4):

$$
\begin{aligned}
& {\left[\begin{array}{cc}
M_{b b}^{\Omega^{+}} & M_{b e}^{\Omega^{+}} \\
M_{e b}^{\Omega^{+}} & M_{e e}^{\Omega^{+}}
\end{array}\right]\left\{\begin{array}{c}
\ddot{u}_{b}^{0} \\
\ddot{u}_{e}^{0}
\end{array}\right\}+\left[\begin{array}{cc}
K_{b b}^{\Omega^{+}} & K_{b e}^{\Omega^{+}} \\
K_{e b}^{\Omega^{+}} & K_{e e}^{\Omega^{+}}
\end{array}\right]\left\{\begin{array}{l}
u_{b}^{0} \\
u_{e}^{0}
\end{array}\right\}} \\
& \quad=\left\{\begin{array}{c}
-P_{b}^{0} \\
P_{e}
\end{array}\right\} .
\end{aligned}
$$

Regarding the consistency of the $\Omega^{+}$domain, the matrices of mass, stiffness, and nodal forces are also unchanged. The nodal force of $P_{e}$ in Eq. (4) can be rewritten in terms of free-field variables at the boundary $\Gamma$ :

$$
P_{e}=M_{e b}^{\Omega^{+}} \ddot{u}_{b}^{0}+M_{e e}^{\Omega^{+}} \ddot{u}_{e}^{0}+K_{e b}^{\Omega^{+}} u_{b}^{0}+K_{e e}^{\Omega^{+}} u_{e}^{0} .
$$

The nodal displacements of the entire domain are obtained by replacing Eq. (5) into Eq. (3). Since Eq. (5)

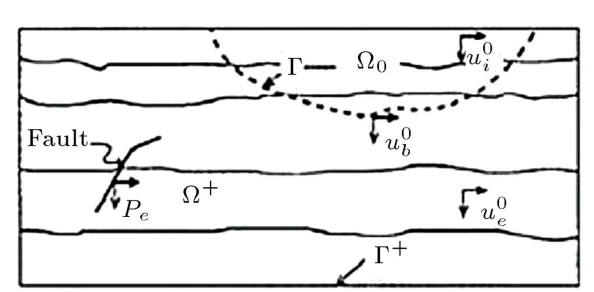

(a)

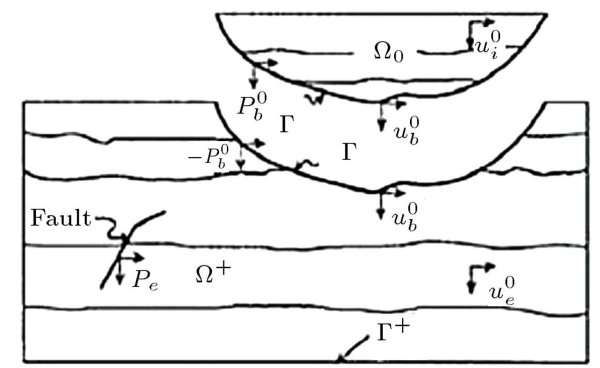

(b)

Figure 4. Auxiliary problem: (a) Entire auxiliary seismic region and (b) auxiliary seismic region partitioned into two subdomains [34]. 
includes $M_{e e}^{\Omega^{+}} \ddot{u}_{e}^{0}$ and $K_{e e}^{\Omega^{+}} u_{e}^{0}$, needing to reserve $u_{e}^{0}$ within the entire domain of $\Omega^{+}$, this formulation does not have any advantages over the usual method. In order to simplify the analysis, a change of variable is recommended. The total displacement of $u_{e}$ is written according to Eq. (6):

$$
u_{e}=u_{e}^{0}+w_{e}
$$

where $w_{e}$ is the relative displacement with respect to $u_{e}^{0}$. By replacing Eq. (6) into Eq. (3), a new equation is achieved:

$$
\begin{gathered}
{\left[\begin{array}{ccc}
M_{i i}^{\Omega} & M_{i b}^{\Omega} & 0 \\
M_{b i}^{\Omega} & M_{b b}^{\Omega}+M_{b b}^{\Omega^{+}} & M_{b e}^{\Omega^{+}} \\
0 & M_{e b}^{\Omega^{+}} & M_{e e}^{\Omega^{+}}
\end{array}\right]\left\{\begin{array}{l}
\ddot{u}_{i} \\
\ddot{u}_{b} \\
\ddot{w}_{e}
\end{array}\right\}} \\
+\left[\begin{array}{ccc}
K_{i i}^{\Omega} & K_{i b}^{\Omega} & 0 \\
K_{b i}^{\Omega} & K_{b b}^{\Omega}+K_{b b}^{\Omega^{+}} & K_{b e}^{\Omega^{+}} \\
0 & K_{e b}^{\Omega^{+}} & K_{e e}^{\Omega^{+}}
\end{array}\right]\left\{\begin{array}{l}
u_{i} \\
u_{b} \\
w_{e}
\end{array}\right\} \\
\quad=\left\{\begin{array}{c}
-M_{b e}^{\Omega^{+}} \ddot{u}_{e}^{0}-K_{b e}^{\Omega^{+}} u_{e}^{0} \\
P_{e}-M_{e e}^{\Omega^{+}} \ddot{u}_{e}^{0}-K_{e e}^{\Omega^{+}} u_{e}^{0}
\end{array}\right\} .
\end{gathered}
$$

Finally, by replacing Eq. (5) into Eq. (7), the final equation is achieved:

$$
\begin{gathered}
{\left[\begin{array}{ccc}
M_{i i}^{\Omega} & M_{i b}^{\Omega} & 0 \\
M_{b i}^{\Omega} & M_{b b}^{\Omega}+M_{b b}^{\Omega^{+}} & M_{b e}^{\Omega^{+}} \\
0 & M_{e b}^{\Omega^{+}} & M_{e e}^{\Omega^{+}}
\end{array}\right]\left\{\begin{array}{l}
\ddot{u}_{i} \\
\ddot{u}_{b} \\
\ddot{w}_{e}
\end{array}\right\}} \\
+\left[\begin{array}{ccc}
K_{i i}^{\Omega} & K_{i b}^{\Omega} & 0 \\
K_{b i}^{\Omega} & K_{b b}^{\Omega}+K_{b b}^{\Omega^{+}} & K_{b e}^{\Omega^{+}} \\
0 & K_{e b}^{\Omega^{+}} & K_{e e}^{\Omega^{+}}
\end{array}\right]\left\{\begin{array}{l}
u_{i} \\
u_{b} \\
w_{e}
\end{array}\right\} \\
\quad=\left\{\begin{array}{c}
0 \\
-M_{b e}^{\Omega^{+}} \ddot{u}_{e}^{0}-K_{b e}^{\Omega^{+}} u_{e}^{0} \\
M_{e b}^{\Omega^{+}} \ddot{u}_{b}^{0}+K_{e b}^{\Omega^{+}} u_{b}^{0}
\end{array}\right\} .
\end{gathered}
$$

The mass and stiffness matrices at the left side of Eq. (8) are same as the matrices in Eq. (3). The force of seismic fault, $P_{e}$, is replaced by the effective nodal forces, according to Eq. (9):

$$
P^{e f f}=\left\{\begin{array}{l}
P_{i}^{e f f} \\
P_{b}^{e f f} \\
P_{e}^{e f f}
\end{array}\right\}=\left\{\begin{array}{c}
0 \\
-M_{b e}^{\Omega^{+}} \ddot{u}_{e}^{0}-K_{b e}^{\Omega^{+}} u_{e}^{0} \\
M_{e b}^{\Omega^{+}} \ddot{u}_{b}^{0}+K_{e b}^{\Omega^{+}} u_{b}^{0}
\end{array}\right\} .
$$

The equation includes sub-matrices of $M_{b e}, K_{b e}, M_{e b}$, and $K_{e b}$, which are mostly zero at all the points of $\Omega^{+}$excluding a finite layer next to $\Gamma$. Hence, the effective force of a finite layer in the auxiliary problem is obtained using Eq. (9). This is the main advantage of Eq. (6).

\subsection{Domain Reduction Method (DRM) algorithm}

The domain reduction method is a two-step procedure, which can be described as follows. In the first step, the problem (Figure 5), including the domains of $\Omega_{0}$ and $\Omega^{+}$, is solved and the values of $u_{e}^{0}$ and $u_{b}^{0}$ are obtained at all nodes of the layer limited to $\Gamma$ and $\Gamma_{e}$ borders. These values are reserved for computing the effective force. Depending on the problem at hand, other methods could be used in order to solve the problem in the first step.

In the second step, the problem is solved at the reduced area of $\Omega \cup \widehat{\Omega}^{+}$including the structure and site (Figure 6). The effective force, which is computed in the first step, is used to solve the problem in the second step; hence, unknowns are calculated. These unknowns are the total displacement field $u_{i}$ in $\Omega, u_{b}$ on $\Gamma$, and the residual displacement field $w_{e}$ in $\widehat{\Omega}^{+}$. Figure 7 presents a flowchart of the DRM process.

\section{Verification of the DRM}

In this section, domain reduction method and its implementation in finite element model are verified. The

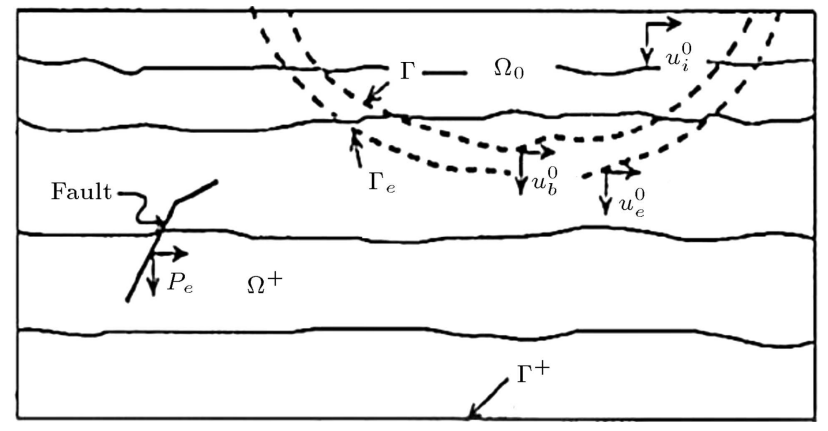

Figure 5. DRM method for a dam system. The first step: The model defines the auxiliary problem over background model [34].

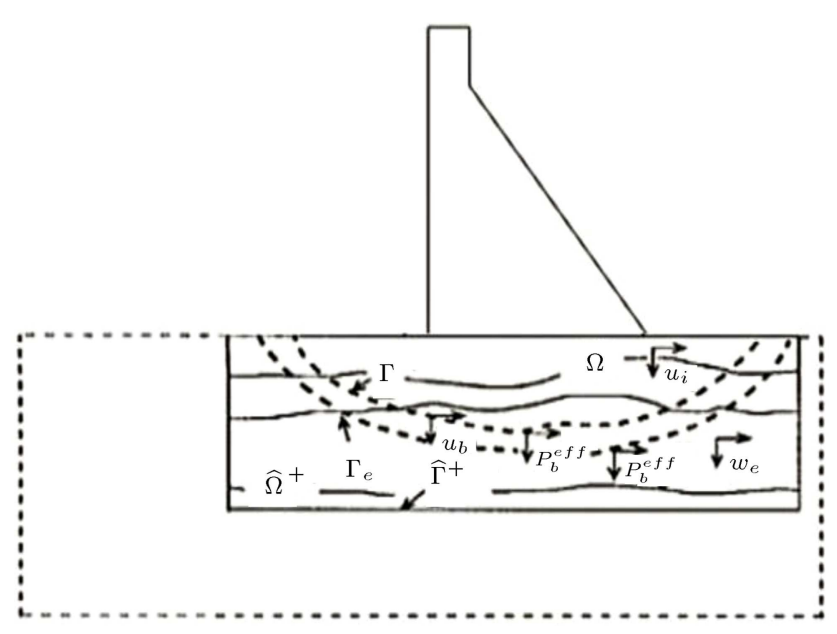

Figure 6. DRM method for a dam system. The second step: The model is defined over the reduced region. 


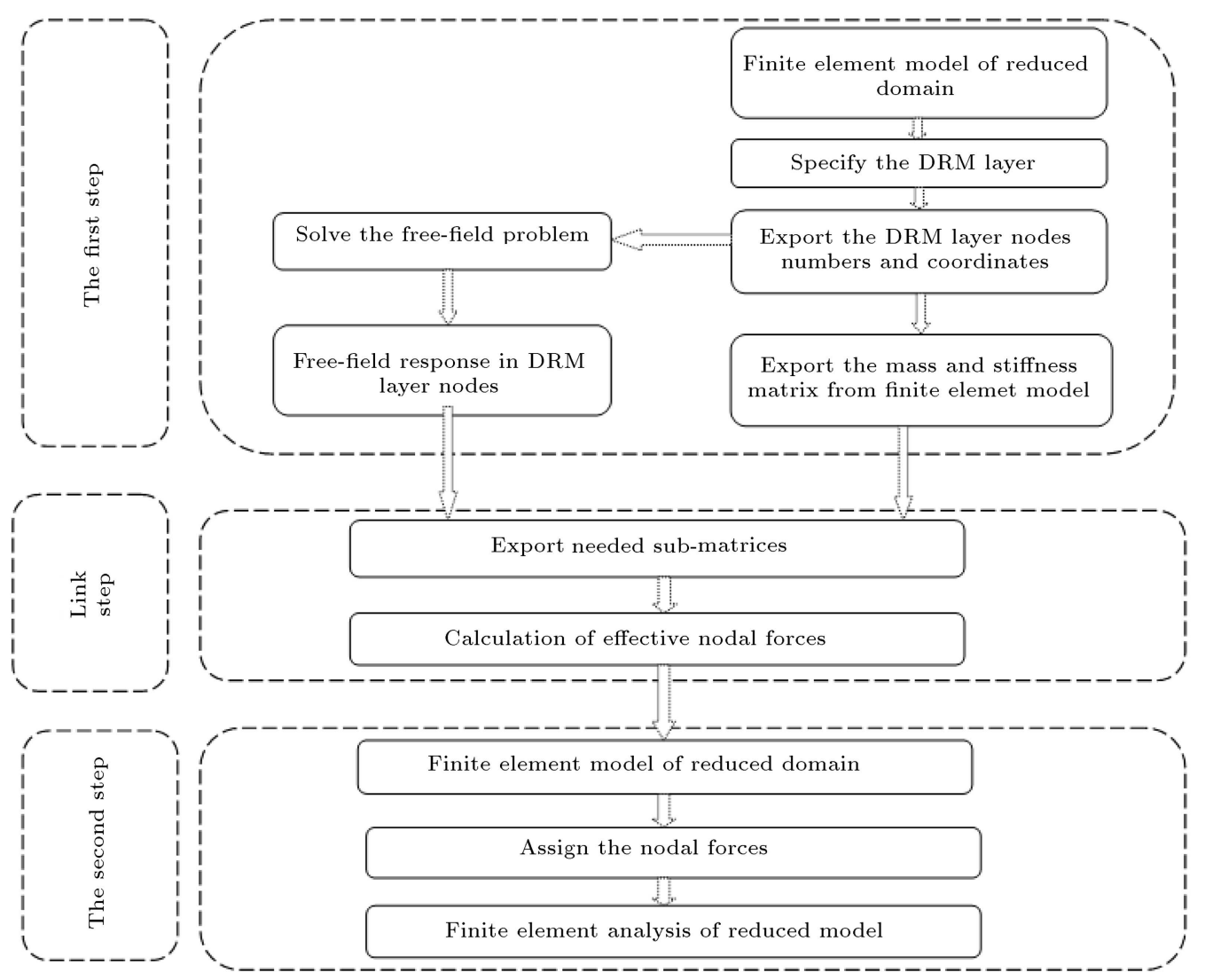

Figure 7. The application process of the DRM.

response in the external region $\widehat{\Omega}^{+}$of the reduced model consists in only outgoing waves corresponding to the existence of the local structures in the reduced model in comparison with the background model. Based on this fact, one way to verify the DRM is to take the interior domain the same in the first and second steps. This is equivalent with the existence of no structure in the model in comparison with the background model. In this case, zero response should be calculated in the external $\widehat{\Omega}^{+}$area of the reduced model. Furthermore, the computed response in the first step should be identical with the response obtained in the second step of the DRM method for the internal areas of $\Omega^{0}$ and $\Omega$.

In summary, by assuming the same material for the interior domain in the first and second steps, the calculated response should be zero in the $\widehat{\Omega}^{+}$domain and same as the result of the first step in the interior $\Omega$ domain. For this purpose, a $2 \mathrm{D}$ half-space domain excited by a point-source fault is considered. For modeling of background model, a 2D large domain is considered for representing the half-space in finite element model that contains the fault. The reduced model is considered a small domain that removes the fault and far-field from background model. The material is assumed to be homogeneous linear elastic with the properties as listed in Table 1. For simplicity, no material damping is considered. Excitation is
Table 1. Material properties.

\begin{tabular}{lc}
\hline Young's modulus $\left(\mathrm{N} / \mathrm{m}^{2}\right)$ & $1940.4 \mathrm{e} 6$ \\
Poisson's ratio & 0.1 \\
Density $\left(\mathrm{kg} / \mathrm{m}^{3}\right)$ & 1800 \\
Shear wave velocity $(\mathrm{m} / \mathrm{s})$ & 700 \\
\hline
\end{tabular}

prescribed as a point source. The responses of two points in interior and exterior domains are compared for verification.

\subsection{First-step model}

A model with dimensions of $10000 \mathrm{~m} \times 5000 \mathrm{~m}$ is considered for simulating the first step of the DRM as shown in Figure 8. This model consists in 20000 fournode quadrilateral elements. The optimum mesh size in an FE model is determined based on the accuracy and computational cost. The mesh size in the wave propagation problems is affected by frequency content of the excitation. Kuhlemeyer and Lysmer [37] showed that for an accurate simulation of wave propagation in an FE analysis, element sizes should be smaller than approximately one-tenth to one-eighth of the shortest wavelength. Based on this fact, the element size in the FE mesh was chosen to be $50 \mathrm{~m}$.

The source of earthquake motions to be used in this model is the time history of a vertical force field. 


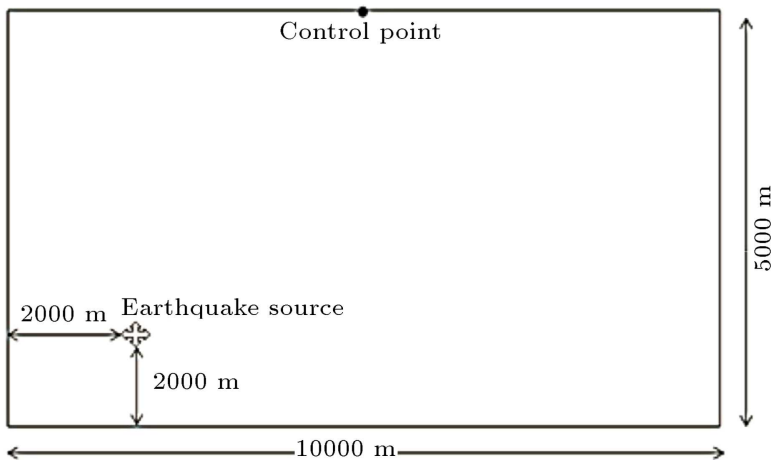

Figure 8. Verification of the DRM; the first-step model.

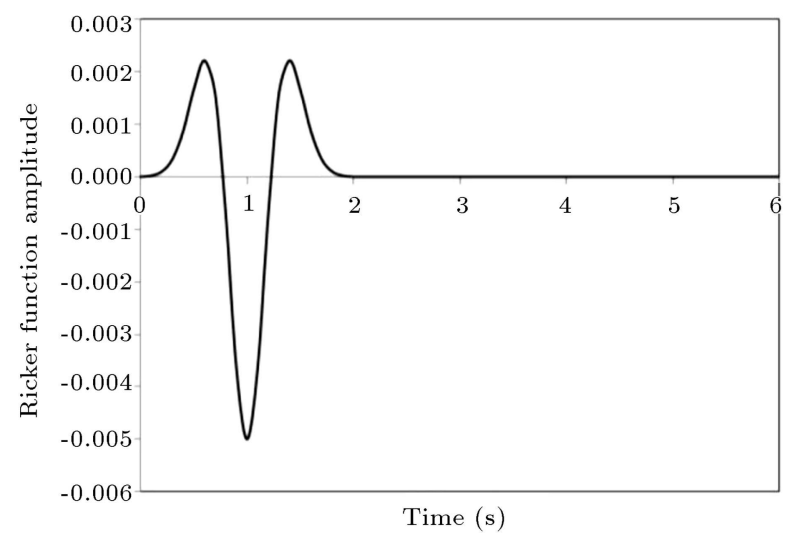

Figure 9. Ricker wavelet used for seismic source.

The source is Ricker wavelet located at $(x=2000 \mathrm{~m}, y=$ $2000 \mathrm{~m})$. The Ricker wavelet formulations are shown in Eq. (10) [38,39]:

$$
R(t)=A\left(2 \pi^{2} \frac{\left(t-t_{s}\right)^{2}}{t_{p}{ }^{2}}-1\right) \exp \left(-\pi^{2} \frac{\left(t-t_{s}\right)^{2}}{t_{p}^{2}}\right),
$$

where $A$ is the maximum amplitude, $t_{s}$ is the time of maximum amplitude, and $t_{p}$ is the main period of the wavelet. Figure 9 shows the displacement time history of Ricker wavelet with dominant frequency of $1 \mathrm{~Hz}$ and maximum amplitude occurring at 1 second.

To implement the boundary condition in the background model, vertical and horizontal displacements were restricted along the truncated sides of the model and horizontal displacements were restricted along the bottom.

\subsection{Second-step model}

A model with the dimensions of $240 \mathrm{~m} \times 70 \mathrm{~m}$ is considered for analysis of the second step as shown in Figure 10. This model consists in 672 four-node quadrilateral elements. The element size in the FE mesh based on the shortest wavelength is chosen to be $5 \mathrm{~m}$. This model is used for verification analysis; therefore, based on the above description, the properties of the model are the same as those of the first model. The

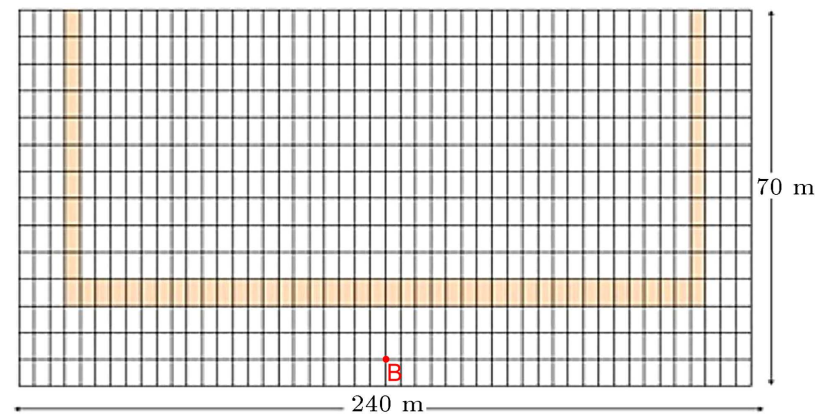

Figure 10. Verification of the DRM; the reduced model.

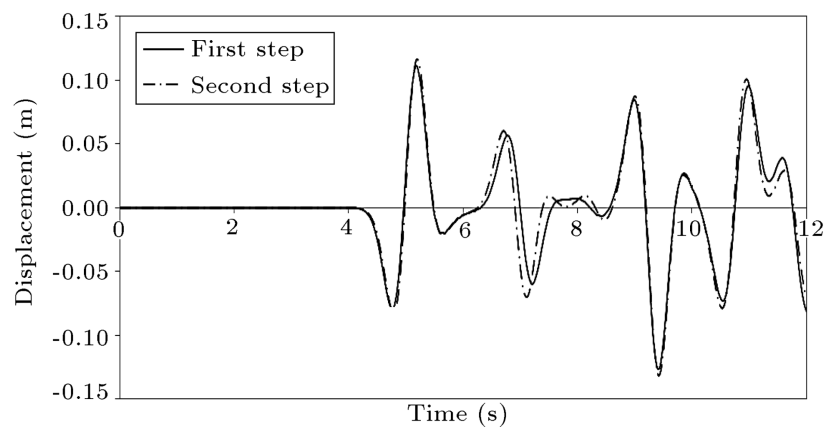

Figure 11. Horizontal component of displacement at control point in the first and second steps in verification analysis.

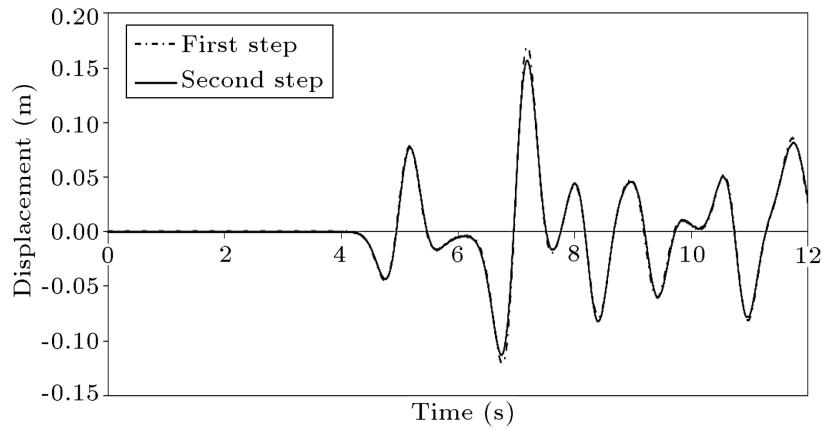

Figure 12. Vertical component of displacement at control point in the first and second steps in verification analysis.

shaded area represents the DRM layer that is the zone of elements between the boundaries $\Gamma$ and $\Gamma_{e}$, in which the effective forces from the first model will be applied.

\subsection{Results}

Displacement time histories at the two points of the model are discussed here between the first- and secondstep models. Comparisons of horizontal and vertical displacements of control point (Figure 8) are shown in Figures 11 and 12. As it can be observed, the results of the second-step model (reduced model) perfectly match those obtained for the first-step model (extended model) in the interior domain points. Figures 13 and 14 are the horizontal and vertical displacement time histories of point B (located outside the DRM layer) in the first and second steps. As mentioned before in 


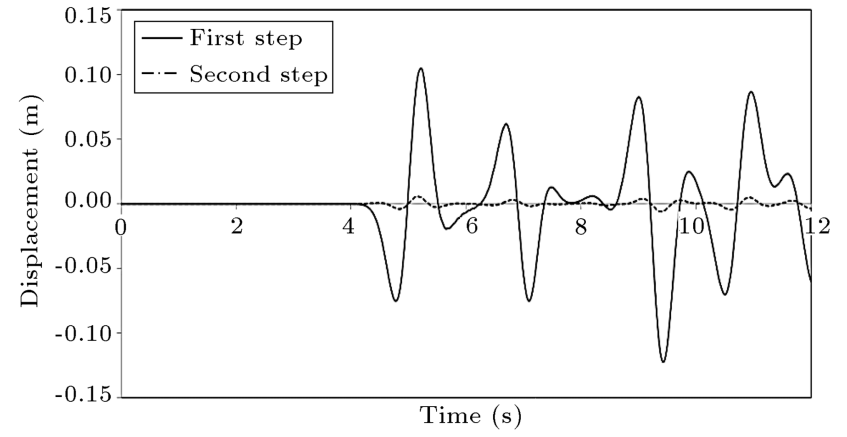

Figure 13. Comparison of horizontal component of displacement at point $\mathrm{B}$ in the first and second steps in verification analysis.

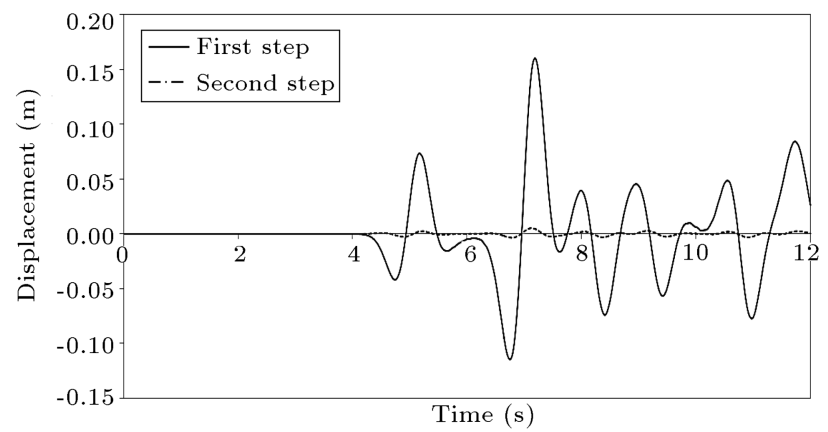

Figure 14. Comparison of vertical component of displacement at point $B$ in the first and second steps in verification analysis.

definition of the DRM, no motion should come out of the DRM layer in case of free field. As shown in these figures, displacement time history at this point is zero in the second step, while it has non-zero value in the first step. The above observation and results verify the domain reduction method and its implementation in finite element model.

\section{DRM application in DAM-foundation-fault system}

In this section, a dynamic analysis is performed to investigate the DRM as an appropriate method in seismic analysis of dam-foundation-fault system. The Koyna concrete gravity dam is selected for case study. An FE analysis is performed for the Koyna dam in two cases. In the first case, an extended foundation with seismic source is used for the purpose of comparison with the second case of a reduced foundation by the use of DRM.

Koyna dam is a concrete gravity dam located in India. In 1963, the construction work of the Koyna dam was finished. The earthquake that happened in the December of 1967, 4 years after its construction, damaged the dam and forced engineers to reassess the dam $[40,41]$.

The tallest section of this dam and the geome-

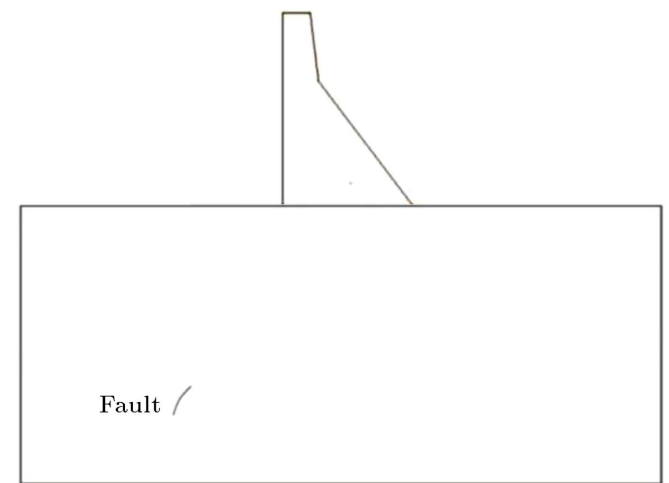

Figure 15. Geometry of the Koyna dam-fault-foundation system.

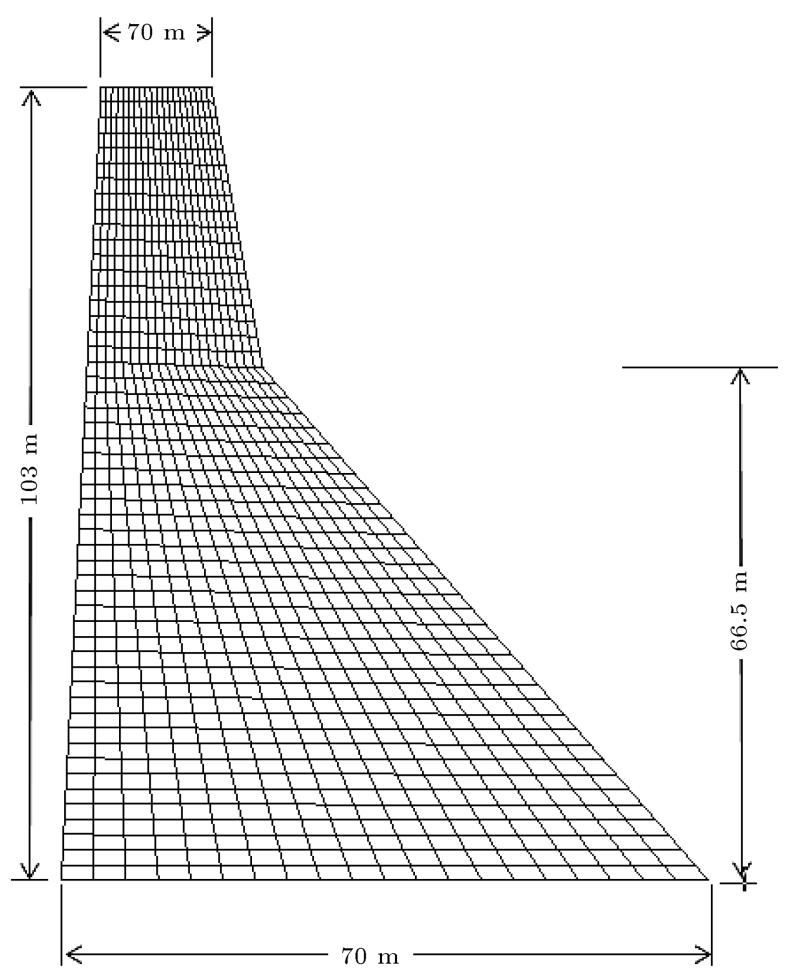

Figure 16. The FE model of the Koyna dam.

try of the dam-fault-foundation system are shown in Figures 15 and 16 . The foundation is modeled same as the first step of the verification model. Figures are not drawn to scale. The modulus of elasticity, mass density, and Poisson's ratio of the dam are taken to be $30000 \mathrm{MPa}, 2630 \mathrm{~kg} / \mathrm{m}^{3}$, and 0.2 , respectively. The dam and foundation are modeled with four-node quadrillateral iso-parametric elements. The dam as well as the foundation is in the state of plane stress. The dynamic analysis is performed by applying Ricker wavelet as a point source fault model at the $(x=2000$ $\mathrm{m} ; y=2000 \mathrm{~m}$ ) point.

\subsection{Koyna dam analysis}

As mentioned above, Koyna dam is analyzed in two cases. The differences of these two cases are in founda- 


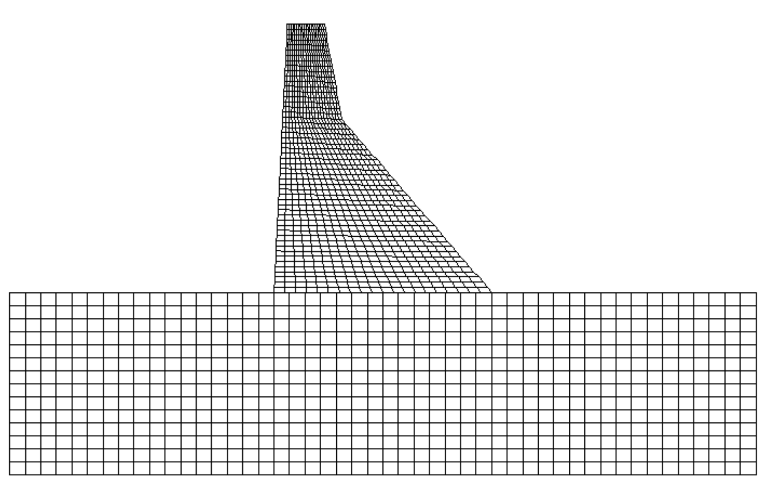

Figure 17. FE model of dam-foundation-fault system in the second step of the DRM.

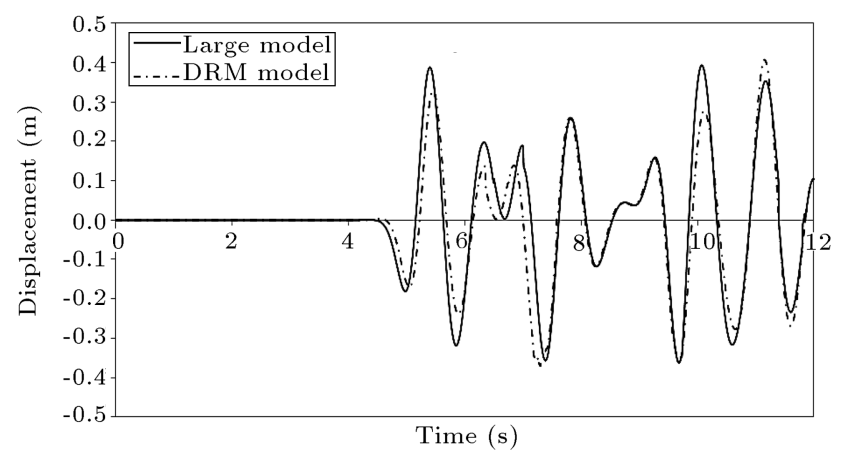

Figure 18. Comparison of horizontal components of displacement at dam crest in the DRM and large (extended) models.

tion modeling and excitation input. In the first case, the extended foundation with large size and the fault are modeled simultaneously. Foundation size in this case is $10000 \mathrm{~m} \times 5000 \mathrm{~m}$. In other words, the first case uses the direct approach. In the second case, the DRM is used as a hybrid approach. The far field and the fault are removed and the foundation size is $240 \mathrm{~m} \times 70 \mathrm{~m}$. The FE model of the Koyna damfoundation-fault system in the second case is shown in Figure 17. The size of the time step in the dynamic part of all analyses is $\Delta t=0.01 \mathrm{sec}$. For comparison, the traditional model consists in 2001040 elements and requires approximately $7 \mathrm{hr}$ for using a system with a Core 2 Duo processor, in contrast to the DRM model, which consists in 1712 elements and takes $4 \mathrm{~min}$ for analysis with the same system and the same processor. The reduction in the required number of mesh elements and analysis time is substantial. This fact translates into considerable computing efficiency, especially for the large-scale problems by considering material nonlinearity.

Figures 18 and 19 show the comparison of horizontal and vertical components of displacement at the crest of dam in the DRM (hybrid) and extended models (direct). As expected, the results of two models have good agreement. Figure 20 shows snapshots of the deformed mesh of the reduced model in DRM case. Displace-

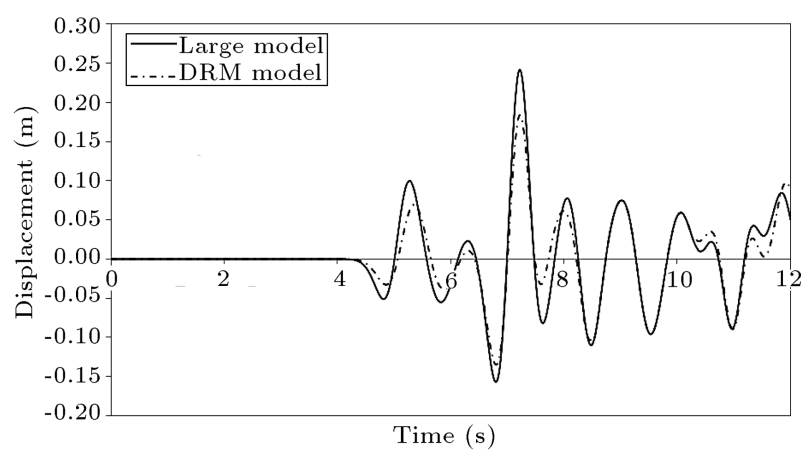

Figure 19. Comparison of vertical components of displacement at dam crest in the DRM and large (extended) models.
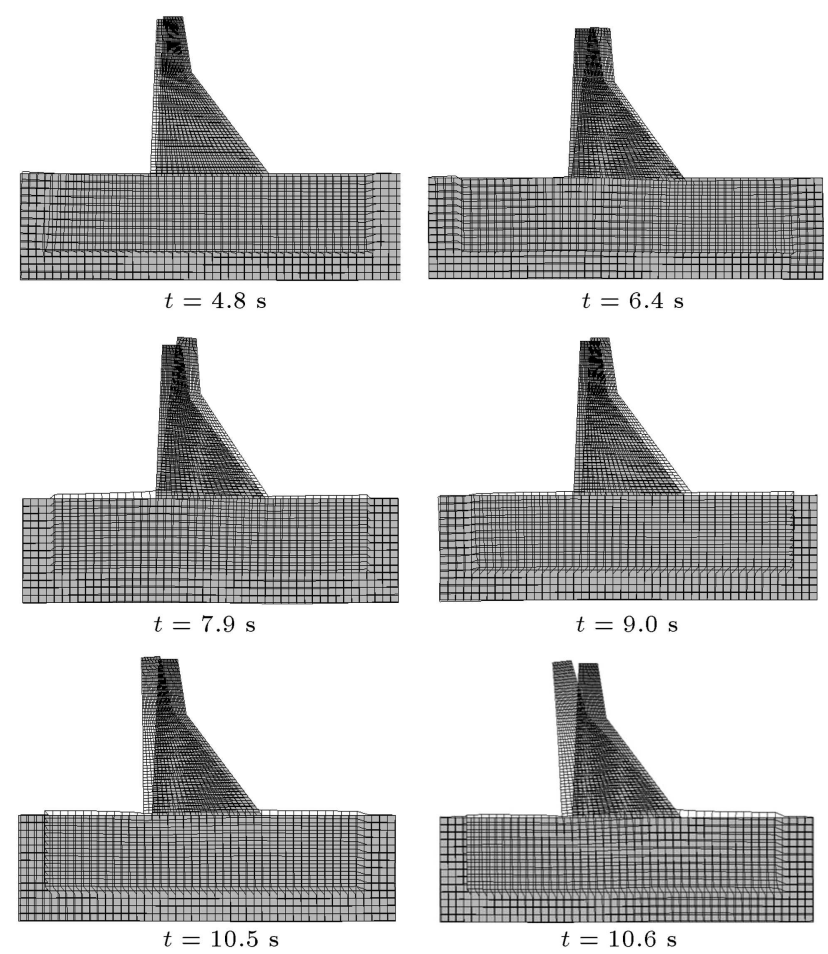

Figure 20. Snapshots of the deformed mesh of the reduced model.

ments are total in interior domain of reduced model, whereas in the exterior domain, they are relative to displacements corresponding to the background model. Since the interior domain is the same in background (extended) and reduced models (i.e., the DRM), the relative response is expected to be very negligible. As can be seen in this figure, the interior domain is deformed at different times, while this is not observed for the exterior domain. These observations demonstrate the very negligible displacement in the exterior domain.

\section{Conclusion}

The DRM is an appropriate two-step procedure for reducing the computational domains in large-scale seis- 
mic analysis by a change of governing variables. This method provides an efficient and reasonably accurate methodology for considering the earthquake source, wave propagation paths, and local site effects.

In the first step of the DRM, a simplified background model (including the source of excitation and excluding the local site effects and dam structure) is analyzed for simulating the earthquake source and propagation path effects. The second step is performed on a reduced domain for modeling the local site effects. Effective nodal forces, derived from the first step, are applied in a single layer of elements in this step.

The implementation of the DRM in FE software was verified for a simple 2D linear model subjected to Ricker wavelet. For this purpose, the internal area of the background and the reduced model were taken to be the same. Thus, zero response was calculated in the external area of the reduced model in the second step analysis. Furthermore, the computed responses for the internal areas in two steps were found to be identical.

In the last part of the paper, to study the DRM as an appropriate method for seismic analysis of damfoundation-fault system, the FE model of the Koyna dam was examined with both the DRM and the traditional approaches. What can be drawn from the results are:

1. In terms of computational costs such as running time, number of elements, and computer system requirements (i.e., CPU and RAM), the DRM is an effective and suitable method versus traditional methods;

2. Assigning artificial boundary to FE models is more comfortable in the DRM, because seismic excitation is directly introduced into the computational domain;

3. Artificial boundary in the DRM is only needed to absorb the scattered energy of the system;

4. The DRM is also a useful tool for soil-structure interaction problems in which the modeling of the fault and propagation path is neglected. Although the linear elastic systems without any damping were illustrated in this study, this methodology can be applied to linear and nonlinear dam-foundationreservoir-fault systems, which will be assessed in future papers.

\section{References}

1. Westergaard, H.M. "Water pressure on dams during earthquakes", Transactions, American Society of Civil Engineers, 1835, pp. 418-433 (1931).

2. Fenves, G. and Chopra, A.K. "Earthquake analysis of concrete gravity dams including reservoir bottom absorption and dam-water-foundation rock interac- tion", Earthquake Engineering \& Structural Dynamics, 12(5), pp. 663-680 (1984).

3. Fenves, G. and Chopra, A.K. "Simplified earthquake analysis of concrete gravity dams", Journal of Structural Engineering, 113(8), pp. 1688-1708 (1987).

4. Ghaemian, M. and Ghobarah, A. "Staggered solution schemes for dam-reservoir interaction", Journal of Fluids and Structures, 12(7), pp. 933-948 (1998).

5. Ghaemian, M. and Ghobarah, A. "Nonlinear seismic response of concrete gravity dams with dam-reservoir interaction", Engineering Structures, 21(4), pp. 306315 (1999).

6. Aghajanzadeh, M. and Ghaemian, M. "Nonlinear dynamic analysis of a concrete gravity dam considering an elastoplastic constitutive model for the foundation", Scientia Iranica, 20(6), pp. 1676-1684 (2013).

7. Mirzabozorg, H. and Ghaemian, M. "Non linear behavior of mass concrete in three dimensional problems using a smeared crack approach", Earthquake Engineering \& Structural Dynamics, 34(3), pp. 247-269 (2005).

8. Lysmer, J. and Kuhlemeyer, R. "Finite element model for infinite media", Journal of Engineering Mechanics, 95, pp. 859-877 (1969).

9. Givoli, D. "High-order local non-reflecting boundary conditions: a review", Wave Motion, 39, pp. 319-326 (2004).

10. Tsynkov, S.V. "Numerical solution of problems on unbounded domains. A review", Applied Numerical Mathematics, 27(4), pp. 465-532 (1998).

11. Basu, U. and Chopra, A.K. "Perfectly matched layers for time-harmonic elastodynamics of unbounded domains: theory and finite-element implementation", Computer Methods in Applied Mechanics and Engineering, 192, pp. 1337-1375 (2003).

12. Basu, U. and Chopra, A.K. "Perfectly matched layers for transient elastodynamics of unbounded domains", International Journal for Numerical Methods in Engineering, 59, pp. 1039-1074 (2004).

13. Bettess, P. "Infinite elements", International Journal for Numerical Methods in Engineering, 11, pp. 53-64 (1977).

14. Léger, P. and Boughoufalah, M. "Earthquake input mechanisms for time-domain analysis of damfoundation systems", Engineering Structures, 11, pp. 37-46 (1989).

15. Clough, R.W. "Non-linear mechanisms in the seismic response of arch dams", Proceedings of the International Conference on Earthquake Engineering, Skopje, Yugoslavia (1980).

16. Tan, H. and Chopra, A.K. "Earthquake analysis of arch dams including dam-water foundation rock interaction", Earthquake Engineering and Structural Dynamics, 24, pp. 1453-1474 (1995). 
17. Chopra, A.K. "Earthquake analysis of arch dams: factors to be considered", Proceedings of the 14th World Conference on Earthquake Engineering, Beijing, China (2008).

18. Kianoush, M.R. and Ghaemmaghami, A.R. "The effect of earthquake frequency content on the seismic behavior of concrete rectangular liquid tanks using the finite element method incorporating soil-structure interaction", Engineering Structures, 33(7), pp. 21862200 (2011).

19. Cakir, T. "Evaluation of the effect of earthquake frequency content on seismic behavior of cantilever retaining wall including soil-structure interaction", Soil Dynamics and Earthquake Engineering, 45, pp. 96-111 (2013).

20. Raghunandan, M. and Abbie, B.L. "Effect of ground motion duration on earthquake-induced structural collapse", Structural Safety, 41, pp. 119-133 (2013).

21. Wang, G., Zhang, S., and Zhou, C. "Correlation between strong motion durations and damage measures of concrete gravity dams", Soil Dynamics and Earthquake Engineering, 69, pp. 148-162 (2015).

22. Li, Y., Conte, J.P., and Barbato, M. "Influence of time-varying frequency content in earthquake ground motions on seismic response of linear elastic systems", Earthquake Engineering \& Structural Dynamics, 45(8), pp. 1271-1291 (2016).

23. Zafarani, H., Noorzad, A., Ansari, A., and Bargi, K. "Stochastic modeling of Iranian earthquakes and estimation of ground motion for future earthquakes in Greater Tehran", Soil Dynamics and Earthquake Engineering, 29(4), pp. 722-741 (2009).

24. Zafarani, H. and Soghrat, M. "Simulation of ground motion in the Zagros region of Iran using the specific barrier model and the stochastic method", Bulletin of the Seismological Society of America, 102(5), pp. 20312045 (2012).

25. Soghrat, M.R., Khaji, N., and Zafarani, H. "Simulation of strong ground motion in northern Iran using the specific barrier model", Geophysical Journal International, 188(2), pp. 645-679 (2012).

26. Mossessian, T.K. and Dravinski, M. "Application of a hybrid method for scattering of $\mathrm{P}, \mathrm{SV}$, and Rayleigh waves by near-surface irregularities", Bulletin of the Seismological Society of America, 77(5), pp. 1784-1803 (1987).

27. Bielak, J., MacCamy, R., McGhee, D., and Barry, A. "Unified symmetric BEM-FEM for site effects on ground motion-SH waves", Journal of Engineering Mechanics, 117(10), pp. 2265-2285 (1991).

28. Fäh, D., Suhadolc, P., Mueller, St., and Panza, G.F. "A hybrid method for the estimation of ground motion in sedimentary basins: Quantitative modeling for Mexico City", Bulletin of the Seismological Society of America, 84(2), pp. 383-399 (1994).

29. Zahradnı'k, J. and Moczo, P. "Hybrid seismic modeling based on discrete-wavenumber and finite-difference methods", Pure and Applied Geophysics, 148(1), pp. 21-38 (1996).

30. Moczo, P., Bystricky', E., Kristek, J., Carcione, J.M., and Bouchon, M. "Hybrid modeling of P-SV seismic motion at inhomogeneous viscoelastic topographic structures", Bulletin of the Seismological Society of America, 87(5), pp. 1305-1323 (1997).

31. Graves, R.W. "Simulating seismic wave propagation in $3 \mathrm{D}$ elastic media using staggered-grid finitedifferences", Bulletin of the Seismological Society of America, 86(4), pp. 1091-1106 (1996).

32. Faccioli, E., Maggio, F., Paolucci, R., and Quarteroni, A. " $2 \mathrm{D}$ and $3 \mathrm{D}$ elastic wave propagation by a pseudospectral domain decomposition method", Journal of Seismology, 1(3), pp. 237-251 (1997).

33. Bao, H., Bielak, J., Ghattas, O., Kallivokas, L.F., O'Hallaron, D.R., Shewchuk, J.R., and Xu, J. "Large-scale simulation of elastic wave propagation in heterogeneous media on parallel computers", Computer Methods in Applied Mechanics and Engineering, 152(1-2), pp. 85-102 (1998).

34. Bielak, J., Loukakis, K., Hisada, Y., and Yoshimura, C. "Domain Reduction Method for three-dimensional earthquake modeling in localized regions, Part I: Theory", Bulletin of Seismological Society of America, 93(2), pp. 817-824 (2003).

35. Yoshimura, C., Bielak, J., Hisada, Y., and Fernandez, A. "Domain Reduction Method for three-dimensional earthquake modeling in localized regions, Part II: Verification and applications", Bulletin of Seismological Society of America, 93(2), pp. 825-840 (2003).

36. Kontoe, S., Zdravkovic, L., Potts, D.M., and Menkiti, C.O. "Case study on seismic tunnel response", Canadian Geotechnical Journal, 45(12), pp. 1743-1764 (2008).

37. Kuhlemeyer, R.L. and Lysmer, J. " Finite element method accuracy for wave propagation problems", Journal of Soil Mechanics and Foundation Division (ASCE), 99(5), pp. 421-427 (1973) (Technical Note).

38. Ryan, H. "Ricker, Ormsby, Klander, Butterworth - a choice of wavelets", Canadian Society of Exploration Geophysicists Recorder, 19(7), pp. 8-9 (1994).

39. Mavroeidis, G.P. and Papageorgiou, A.S. "A mathematical representation of near fault ground motions", Bulletin of the Seismological Society of America, 93(3), pp. 1099-1131 (2003).

40. Chopra, A.K. and Chakrabarti, P. "The earthquake experience at Koyna dam and stresses in concrete gravity dams", Earthquake Engineering and Structural Dynamics, 1(2), pp. 151-164 (1972).

41. Rajendran, K. and Harish, C.M. "Mechanism of triggered seismicity at Koyna: An evaluation based on relocated earthquakes", Current Science, 79(3), pp. 358-363 (2000). 


\section{Biographies}

Hamid Mohammadnezhad is a researcher in the Civil Engineering Department of Sharif University of Technology, Tehran, Iran. His research interests are in wave propagation, soil-structure-fluid interaction, and seismic analysis of infrastructures (dam, bridge, tunnel, tower, etc.). In recent years, he has been working on linear and nonlinear seismic analysis of concrete dams based on numerical modeling of wave propagation from source to site.

Hamid Zafarani is Associate Professor at Interna- tional Institute of Earthquake Engineering and Seismology (IIEES), Tehran, Iran. His research interests span a wide range of topics including earthquake simulation, hazard analysis, numerical methods, earthquake prediction, and strong motion studies.

Mohsen Ghaemian is Professor in the Civil Engineering Department of Sharif University of Technology, Tehran, Iran. His current research activities include dynamic responses of gravity and arch dams, dam-reservoir-foundation interaction effects, seismic response of dams due to non-uniform excitations, and nonlinear behavior of concrete dams. 